\title{
Foresight is Partially Formed from Resilience: The Relationship Between Self-Esteem and Future Time Perspective Among Japanese High School Students
}

\author{
Yukihiro Takagishi ${ }^{1,}$, , Tomoko Kuraoka ${ }^{2}$, Tomohiro Ide $^{3}$ \\ ${ }^{1}$ Faculty of Education, Kumamoto University, Kumamoto, Japan \\ ${ }^{2}$ Department of Student Counselling, Kumamoto Seiryo High School, Kumamoto, Japan \\ ${ }^{3}$ Faculty of Education, Shizuoka University, Shizuoka, Japan \\ Email address: \\ takagishi@educ.kumamoto-u.ac.jp (Y. Takagishi) \\ *Corresponding author
}

To cite this article:

Yukihiro Takagishi, Tomoko Kuraoka, Tomohiro Ide. Foresight is Partially Formed from Resilience: The Relationship Between Self-Esteem and Future Time Perspective Among Japanese High School Students. International Journal of Education, Culture and Society.

Vol. 2, No. 2, 2017, pp. 76-82. doi: 10.11648/j.ijecs.20170202.15

Received: February 27, 2017; Accepted: March 13, 2017; Published: March 28, 2017

\begin{abstract}
Self-esteem is considered as an influential factor that makes foresight positive; however, people experience a variety of adversities over their lifetime that impair their self-esteem. Nevertheless, many people still have positive foresight because of the influence of resilience, which aid one in overcoming specific adversities. We examined the relationship between self-esteem, future time perspective, and resilience. Japanese high school students $(\mathrm{N}=1427$; boys $=596$, girls $=831)$ completed a questionnaire comprising self-esteem, time perspective, and resilience scales. A series of path analyses demonstrated that self-esteem facilitated future hope and mitigated present feeling of emptiness. Furthermore, resilience mediated the relationship between self-esteem and time perspective. Self-directedness of resilience increased future hope. Additionally, being optimistic could positively influence foresight; however, there were sex differences in its function. Among boys, being optimistic increased future hope; among girls, being optimistic decreased present emptiness. These results suggest that gaining the ability to overcome adversities may affect being positive about one's future. However, youths should be taught how to relax and think positively, not only to enhance future hope, but also to mitigate emptiness.
\end{abstract}

Keywords: Time Perspective, Resilience, Self-Esteem, High School Student

\section{Introduction}

To put a positive spin on one's future and not ruminate on past trauma is important for one's mental health. Especially, it is crucial for youth to consider their future to be positive as they are preparing to enter the work force [1]. The sense of future time perspective (FTP) has been discussed in previous literature. After the term "time perspective" was first used by Frank [2], Lewin [3] defined time perspective as "the totality of the individual's views of his/her psychological past and future existing at a given time (p. 75)." The concept of time perspective includes past, present, and future vison [4]. Shostrom [5] noted that the ability to all three of these leads to self-realization. Future vison should be hopeful and healthy for youth in light of the fact that FTP pertains to academic success, hope for one's future, and present motivation [6-8]. Furthermore, FTP works as a buffer against suicidal behavior [9].

Self-esteem is one of the factors that affects whether one's future vision is positive. Tsuzuki [10] found a relationship between increased hope and self-esteem in one's time perspective during the transition from elementary to junior high school. Marttinen \& Salmela-Aro [11] revealed that high school students who have self-centered goals tend to have low self-esteem. Worrell et al. [12] also reported a relationship between high self-esteem and positive FTP. They found that high self-esteem was negatively correlated with a negative past perspective and a present fatalistic attitude. Feelings toward school achievement are important for youths because they relate to morality development, academic achievement, 
and so on. King and Gaerlan [13] found that FTP contributed to the establishment of joy and hope while studying. On the other hand, Laghi et al. [14] reported that youths who partake in risky behavior such as binge eating and drinking alcohol tend to have poor FTP and low self-esteem.

Not only has empirical research demonstrated that high self-esteem is correlated with a positive time perspective, it can also be discussed from a theoretical viewpoint. People experience a variety of adversities in their lives. The outcomes of these and the way people view the future is influenced by how they deal with them. One of the key concepts in dealing with adversities positively is resilience [15].

Resilience has been explored in relation to recovering from mental disorders and psychological trauma. Rutter [16] first defined resilience as phenomenon to maintain an adaptive mechanism despite serious risks. Since this, other definitions have been proposed; however, two components (i.e., "exposure of adversity" and "achievement of positive adaptation") are essential to resilience [17]. In the risk study for a variety of research areas, resilience is considered to be a protective factor. In fact, many empirical research reported that when people are exposed to serious stress, the more resiliency they have, the more protective power they show [18, 19].

Although resilience as a personal attribution is a protective factor against risk, it does not affect the outcome of the risky events only by its own. Therefore, resilience has been viewed as a process of adaptation [20]. For this reason, if youths decide to get into a delinquent group, through the process of adaptation, resilience could work as a negative accelerator [21]. Namely, an important component is how resilience interacts with related factors and how resilience evolves over time.

Several studies have reported a relationship between resilience and self-esteem [22, 23]. Benetti \& Kambouropouloss [24] found that resilience indirectly raised positive affect and buffered self-esteem. Kidd \& Shahar [25] studied the resilience of homeless youths and revealed that it was an essential component of overcoming social and personal risks and self-esteem acted as a protective factor when youths were challenged with a variety of difficulties. Moreover, Dumont \& Provot [26] speculated that youths' resilience was related to their frequency of using problem-solving coping strategies, and found that resilient youths had higher self-esteem than their non-resilient counterparts. An intention to adopt a problem-solving coping strategy is a behavior that is related to one's future. Therefore, it is likely that the relationship between resilience and self-esteem, could affect FTP. Consequently, we examined whether resilience mediates and facilitates FTP and if it is affected by self-esteem among high school students.

\section{Methods}

\subsection{Participants}

Japanese high school students were recruited $(\mathrm{N}=1427$; boys $=596$, girls $=831)$ from 5 high schools $(1$ st, 2 nd, and 3 rd year students comprised 462, 513, and 452 respectively). While disturbing the questionnaire, the participants were informed about data confidentiality and anonymity. The participants were also told they were free to withdraw at any time without any consequences.

\subsection{Measurements}

\subsubsection{FTP}

The Time Perspective Scale, which Tsuzuki [27] developed, was used in this study. The scale consists of 5 subscales: Hope for the future (5 items, e.g., "I think my future is bright"); Future orientation ( 5 items, e.g., "I often think about when I become adult"); Emptiness (4 items, e.g., "I sometimes feel emptiness every day"); Planning (5 items, e.g., "I am doing things quickly based on the plan"); and Desire for the future (3 items, e.g., "I want to find my future goal"). All 22 items are rated using a 4-point scale ranging from 1 (disagree) to 4 (agree). This study only used "Hope for the future" and "Emptiness" subscales because we focused on whether high school students feel hope or emptiness towards their future.

\subsubsection{Self-Esteem}

The Japanese version of Harter's [28] Perceived Competence Scale [29] was used. The scale comprise 10 items and that are rated using a 4-point scale ranging from 1 (disagree) to 4 (agree) (e.g., "I am confident"). Both Harter [28] and Sakurai [29] confirmed a one-factor structure and the Cronbach's alpha of this study was. 840 .

\subsubsection{Resilience}

The Resilience Scale, which was developed by Ishige et al. [30] was used. The scale comprises 21 items and that are rated using a 4-point scale ranging from 1 (disagree) to 4 (agree). The scale comprises 3 factors: Self-directedness $(\alpha=.851)$, Optimism $(\alpha=.851)$, and Relation-orientedness $(\alpha=.814)$. These factors refer to the intention to cope with problems by oneself through revising one's judgement and behavior, the tendency to think of things positively, and a disposition that emphasizes one's relationship with others to overcome difficulty, respectively.

\subsection{Statistical Analyses}

First, an exploratory factor analysis using the principal factor method and Promax rotation was conducted to confirm the factor structure of the Time Perspective Scale. Second, a series of hierarchical multiple regression analyses were conducted: "Hope for the future" and "Emptiness" were set as dependent variables. Then, path analyses were conducted. The fit of each model with the data was examined with a chi-squared test (CMIN), goodness-of-fit index (GFI), adjusted goodness-of-fit index (AGFI), comparative fit index (CFI), and root mean square error of approximation (RMSEA). Per conventional criteria, a good fit would be indicated by $\mathrm{CMIN} / \mathrm{df}<2$, GFI $>.95$, AGFI $>.85$, CFI $>.95$, and RMSEA $<.08$ [31].

All statistical analyses were conducted using the Statistical 
Package for Social Science (SPSS) version 22.0 and AMOS 22.0.

\section{Results}

\subsection{Factor Structure of the Time Perspective Scale}

A five-factor structure emerged through the exploratory factor analysis for the Time Perspective Scale (i.e., principal factor method and Promax rotation), which was consistent with Tsuzuki's original version [27] including item constitution. We used "Hope for the future $(\alpha=.855)$ " and "Emptiness $(\alpha=.746)$ " factors, which comprised 5 items (e.g., "I think my future is bright" and "I have hope for my future") and 4 items (e.g., "I sometimes feel emptiness every day" and "Every day is boring with same old routine"), respectively for later analyses. The results implied that we should be careful about multicollinearity issues during the multiple regression analysis.

\subsection{Bivariate Statistics}

A correlational analysis was conducted for all subscales (Table 1). We found significant correlations among all combinations. "Hope for the future" was positively correlated with all variables except "Emptiness." "Emptiness" was negatively correlated with all variables except "Hope for the future."

Additionally, sex differences were explored using t-tests. Sex differences existed for "Hope for the future" $(\mathrm{t}(1425)=$ $2.163, \mathrm{p}<.05)$, "Self-esteem" $(\mathrm{t}(1425)=5.298, \mathrm{p}<.01)$, and "Relation-orientedness" $(\mathrm{t}(1425)=8.531, \mathrm{p}<.01)$. Boys' scores for "Hope for the future" and "Self-esteem" were higher than girls' scores were; however, girls' scores for "Relation-orientedness" were higher than boys' scores were.

Table 1. Pearson Correlations, Means, and Standard Deviations of Time perspective, Self-Esteem, and Resilience.

\begin{tabular}{|c|c|c|c|c|c|c|}
\hline & 1 & 2 & 3 & 4 & 5 & 6 \\
\hline 1 Hope for the future & - & & & & & \\
\hline 2 Emptiness & $-.298 * * *$ & - & & & & \\
\hline 3 Self-Esteem & $.572 * * *$ & $-.411 * * *$ & - & & & \\
\hline 4 Self-directedness & $.336^{* * *}$ & $-.154 * * *$ & $.201 * * *$ & - & & \\
\hline 5 Relation-orientedness & $.237 * * *$ & $-.178 * * *$ & $.189^{* * *}$ & $.365^{* * *}$ & - & \\
\hline 6 Optimism & $.438 * * *$ & $-.311 * * *$ & $.509 * *$ & $.272 * * *$ & $.207 * * *$ & - \\
\hline$S D$ & 2.9 & 2.6 & 4.9 & 4.1 & 4.1 & 2.6 \\
\hline
\end{tabular}

*** $p<.001$.

\subsection{Hierarchical Multiple Regression Analyses}

Two hierarchical multiple regression analyses using the forced entry method were conducted. One model was set with "Hope for the future" as the dependent variable; other factors (i.e., step 1: "Self-directedness," "Relation-orientedness" and "Optimism" and step 2: "Self-esteem") were set as independent variables. The other model was set with
"Emptiness" as the dependent variable and the independent variables were the same as the first model. Sex was also entered as a dummy variable. "Self-directedness," "Optimism," and "Self-esteem" all significantly contributed to "Hope for the future" and "Optimism." "Self-esteem" significantly contributed to "Emptiness." All VIFs were $<1.5$; therefore multicollinearity was avoided (Table 2).

Table 2. The result of hierarchical multiple regression analysis.

\begin{tabular}{|c|c|c|c|c|c|c|c|c|}
\hline \multirow{2}{*}{ Model } & \multicolumn{4}{|c|}{ Hope for the future } & \multicolumn{4}{|c|}{ Emptiness } \\
\hline & B & SE & $\beta$ & $p$ & B & SE & $\beta$ & $p$ \\
\hline \multicolumn{9}{|l|}{ Step 1} \\
\hline Sex & .340 & .157 & .057 & .031 & 0.79 & .139 & .015 & .570 \\
\hline \multicolumn{9}{|l|}{ Step 2} \\
\hline Sex & .281 & .141 & .047 & .047 & .080 & .137 & .015 & .560 \\
\hline Self-directedness & .146 & .018 & .203 & .000 & -.020 & .018 & -.032 & .251 \\
\hline Relation-orientedness & .054 & .018 & .075 & .003 & -.032 & .018 & -.050 & .072 \\
\hline Optimism & .405 & .027 & .365 & .000 & -.264 & .026 & -.269 & .000 \\
\hline \multicolumn{9}{|l|}{ Step 3} \\
\hline Sex & .676 & .128 & .114 & .000 & -.185 & .132 & -.035 & .162 \\
\hline Self-directedness & .133 & .016 & .185 & .000 & -.012 & .017 & -.018 & .485 \\
\hline Relation-orientedness & .018 & .017 & .024 & .287 & -.008 & .017 & -.012 & .651 \\
\hline Optimism & .165 & .027 & .148 & .000 & -.103 & .028 & -.105 & .000 \\
\hline Self-esteem & .278 & .015 & .464 & .000 & -.186 & .015 & -.352 & .000 \\
\hline
\end{tabular}

\subsection{Structural Equation Model}

Based on the result of multiple regression analyses and our theoretical framework, a path model was built where "Hope for the future" and "Emptiness" were set as dependent variables. Resilience mediated the causal paths set from "Self-esteem" for both outcomes.

After deleting non-significant paths, the path analysis was 
repeated for the best fit model. The final model, as indicated in

Figure 1 , had acceptable values $(\mathrm{CMIN}=9.526$, GFI $=.998$,

$\mathrm{AGFI}=.988, \mathrm{CFI}=.997$, RMSEA $=.031)$.

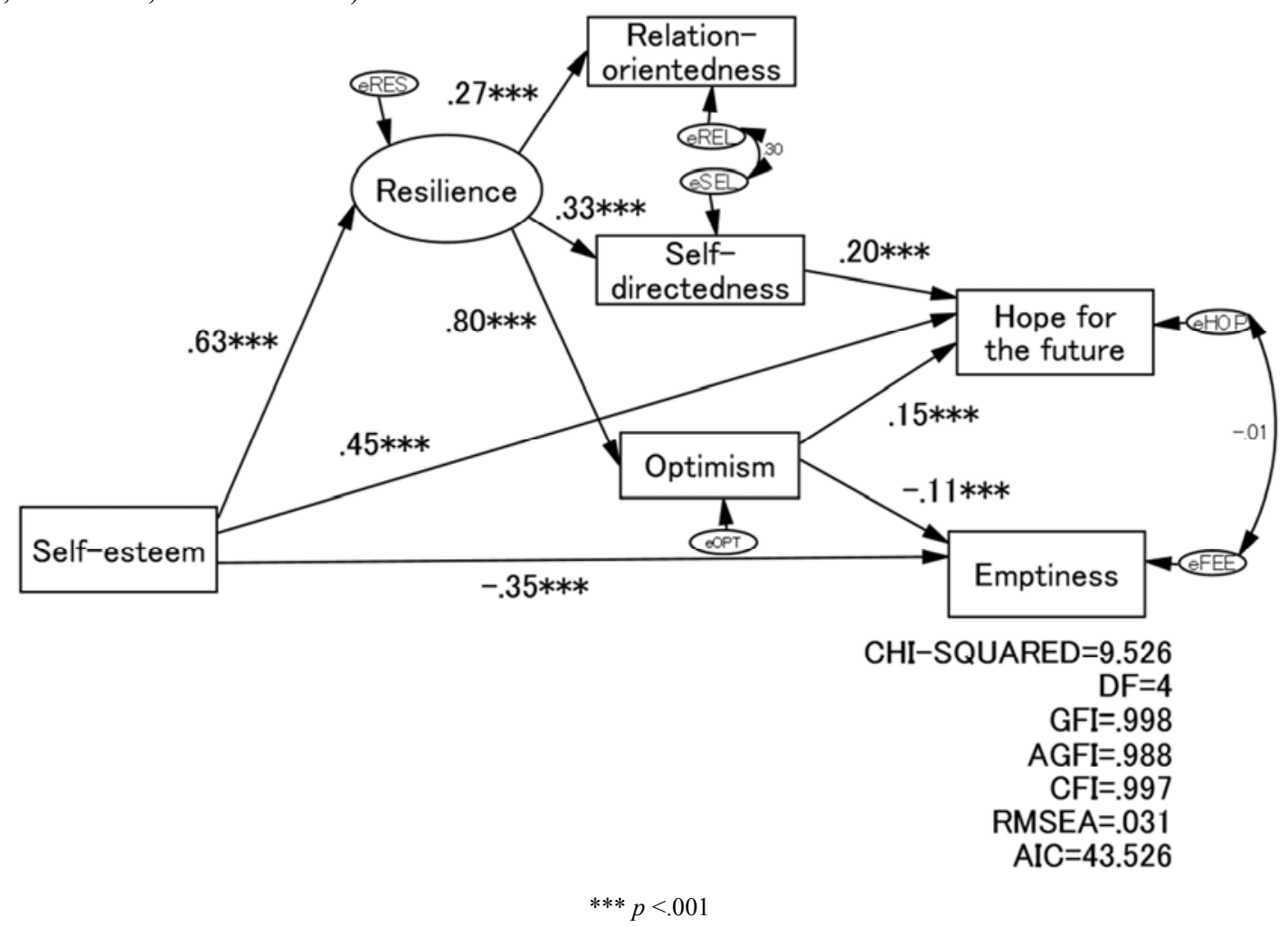

Figure 1. Moderator role of resilience in relationship between self-esteem and future time perspectives.

i. Simultaneous multiple-group analysis

To examine sex differences among some variables, we compared boys and girls separately using a simultaneous multiple-group analysis. The results showed that, in the model that no-restraint was set for, every parameter demonstrated a better fit than the one that every parameter was restricted to be the same across the two corresponding groups, indicating heterogeneity of both models (GFI $=.996$, AGFI $=.977$, CFI $=.994$, $\mathrm{RMSEA}=.031, \mathrm{AIC}=86.782$; and GFI $=.980$, AGFI $=.938, \mathrm{CFI}=.929, \mathrm{RMSEA}=.045, \mathrm{AIC}=96.786$, respectively).
Consequently, a path analysis was conducted from the first model (Figure 1) for each sex. Non-significant paths were deleted to explore a better fit in the analysis. The path from "Optimism" to "Emptiness" was deleted in the boys' group; the path from "Hope for the future" was deleted in the girls' group. Furthermore, a pairwise comparison of parameters showed that the path coefficient from "Resilience" to "Relation-orientedness" in the girls' group was significantly higher than it was in the boys' group. Each structure of the path model is indicated in Figures 2 and 3.

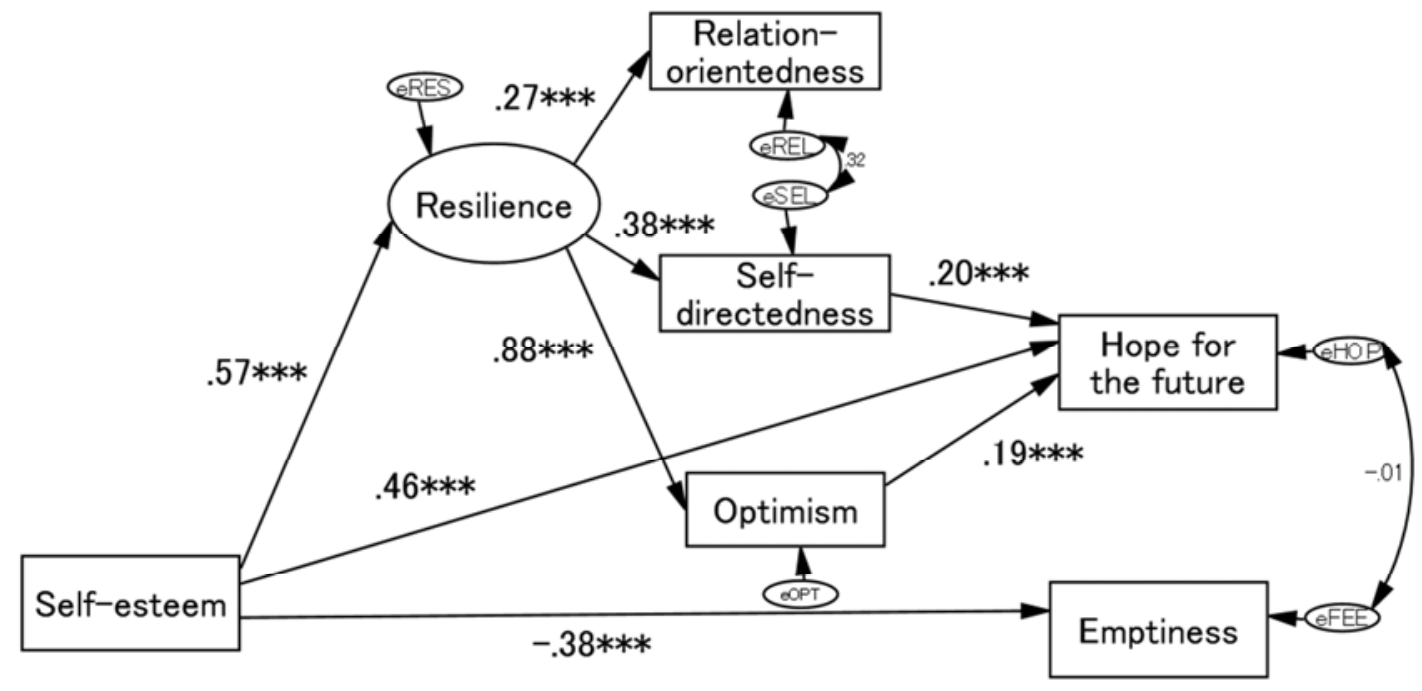


$* * * p<.001$

Figure 2. Men's group model.

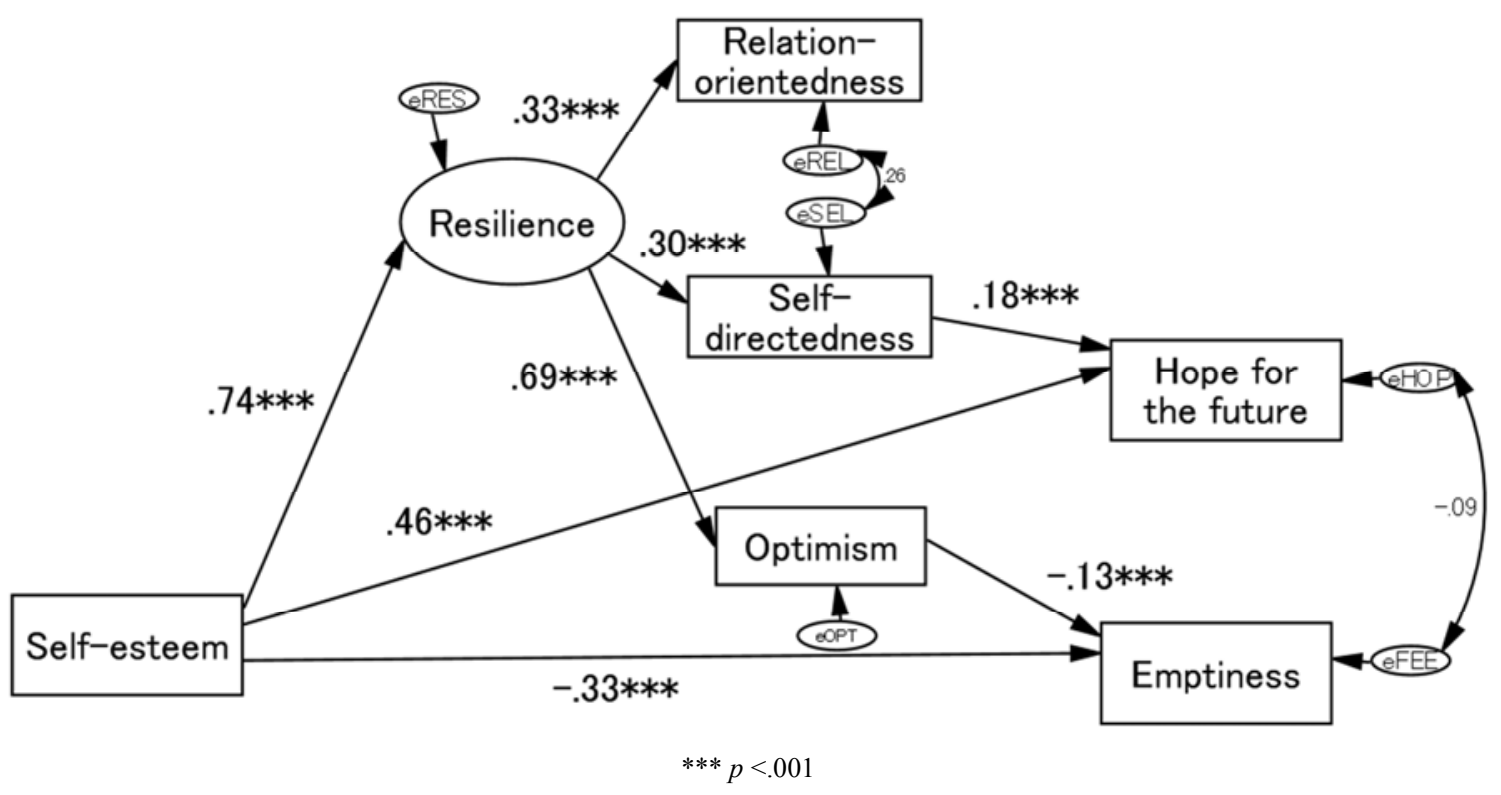

Figure 3. Women's group model.

\section{Discussion}

We examined whether resilience mediates the relationship between self-esteem and FTP and if resilience buffers the degree of FTP.

A path analysis demonstrated that self-esteem directly facilitated one's hope for the future and mitigated one's emptiness. Additionally, resilience mediated the relationship between self-esteem and FTP. In particular, optimism and self-esteem facilitated one's hope for the future and weakened one's emptiness. Resilience refers to the ability and attitude one displays when faced with difficulties. Whereas two subscales of the resilience scale (i.e., "Self-directedness" and "Relation-orientedness") represent active and assertive behavior, optimism refers to passive behavior such as not worrying and not sulking. Therefore, since it is crucial for youth to have hope for their future, they should be guided on how to relax, think positively, and eagerly attempt new things.

Simultaneous multiple group analyses showed heterogeneity of the path models between both sex groups. Optimism affected hope for the future among boys' and optimism affected emptiness among girls. Even though having hope for the future is important, Kasho [32] noted that people are not motivated by only focusing on their future. In addition, the present work shows that girls' hope for the future was also higher than boys' one. It is understandable because girls have causal pass to make present satisfactory by being optimistic. Also, in light of gender role, boys are expected to set a higher goal by social factor such as their family and teachers and so on. On the other hand, girls are considered that they give greater importance to peer relationship than boys do [33, 34]. Because focusing on one's present makes him/her being motivated, further research should clarify why high school male students view their future positively.

Many previous researchers have reported that men's self-esteem is higher than women's [35-39]. Our results consistently revealed that boys' self-esteem was higher than girls' self-esteem was. Previous research suggested this is related to gender roles. This could be true for this study as gender roles have typically been internalized by the time individuals are in high school. Meanwhile, since youths' self-esteem is still "under construction" [40], we should pay attention to changes in FTP that coincide with changes in self-esteem among youths.

Furthermore, "Relation-orientedness" was higher for girls as opposed to boys. This result could be explained by social tendencies as women tend to depend on others when they are in stressful situations more so than men do. In fact, among those poor mental health, men less frequently seek help from mental health professionals such as psychiatrists and counselors than women do [41]. This is important since seeking help from other people is a component of resilience.

Two limitations of this study should be noted. First, we used a cross-sectional design; therefore, further research needs to implement a longitudinal design to examine causality. Second, although we focused on FTP, additional studies should examine past and present time perspective simultaneously to clarify the structure of FTP in more detail.

\section{Conclusion}

This study revealed that resilience mediated the relationship between self-esteem and FTP. Additionally, being optimistic could positively influence foresight; however, there were sex 
differences in its function. Among boys, being optimistic increased future hope; among girls, being optimistic decreased present emptiness. These results suggest that gaining the ability to overcome adversities may affect being positive about one's future. However, youths should be taught how to relax and think positively, not only to enhance future hope, but also to mitigate emptiness.

\section{References}

[1] Kerpelman, J. L., \& Mosher, L. S. (2004). Rural African American adolescents' future orientation: The importance of self-efficacy, control and responsibility, and identity development. Identity, 4, 187-208.

[2] Frank, L. K. (1939). Time perspectives. Journal of Social Philosophy, 4, 293-312.

[3] Lewin, K. (1951). Field theory in social science: Selected theoretical papers. New York: Harper \& Row.

[4] Zimbardo, P. G., \& Boyd, J. N. (2015). Putting time in perspective: A valid, reliable individual-differences metric. Journal of Personality and Social Psychology, 77, 17-55.

[5] Shostrom, E. L. (1968). Time as an integrating factor. In C. Buhler \& F. Massarik (Eds.), The course of human life. A study of goals in the humanistic perspective. New York: Springer, 351-359.

[6] Nuttin, J. R. (1964). The future time perspective in human motivation and learning. Acta psychologica, 23, 60-82.

[7] De Volder, M. L., \& Lens, W. (1982). Academic achievement and future time perspective as a cognitive-motivational concept. Journal of Personality and Social Psychology, 42, 566-571.

[8] Oyserman, D., Bybee, D., \& Terry, K. (2006). Possible selves and academic outcomes: How and when possible selves impel action. Journal of personality and social psychology, 91, 188-204.

[9] Chin, J., \& Holden, R. R. (2013). Multidimensional future time perspective as moderators of the relationships between suicide motivation, preparation, and its predictors. Suicide and Life-Threatening Behavior, 43, 395-405.

[10] Tsuzuki, M. (2012). Dynamic changing process of hope in early adolescence: Analysis of individual differences during the transition from elementary school to junior high school1. Japanese Psychological Research, 54, 253-262.

[11] Marttinen, E., \& Salmela-Aro, K. (2012). Personal goal orientations and subjective well-being of adolescents. Japanese Psychological Research, 54, 263-273.

[12] Worrell, F. C., McKay, M. T., \& Andretta, J. R. (2015). Concurrent validity of Zimbardo Time Perspective Inventory profiles: A secondary analysis of data from the United Kingdom. Journal of adolescence, 42, 128-139.

[13] King, R. B., \& Gaerlan, M. J. M. (2014). How you perceive time matters for how you feel in school: Investigating the link between time perspectives and academic emotions. Current Psychology, 33, 282-300.

[14] Laghi, F., Liga, F., Baumgartner, E., \& Baiocco, R. (2012).
Time perspective and psychosocial positive functioning among Italian adolescents who binge eat and drink. Journal of adolescence, 35, 1277-1284.

[15] Masten, A. S., \& Reed, M. J. (2002). Resilience and development. [In] CR Snyder SJ López (Eds.), Handbook of Positive Psychology (pp. 74-88). New York: Oxford University Press.

[16] Rutter, M. (1985). Resilience in the face of adversity. British Journal of Psychiatry, 147, 598-611.

[17] Luthar, S. S., Cicchetti, D., \& Becker. B. (2000). The construct of resilience: A critical evaluation and guidelines for future work. Child Development, 71, 543-562.

[18] Boyce Rodgers, K., \& Rose, H. A. (2002). Risk and resiliency factors among adolescents who experience marital transitions. Journal of Marriage and Family, 64, 1024-1037.

[19] Buckner, J. C., Mezzacappa, E., \& Beardslee, W. R. (2003). Characteristics of resilient youths living in poverty: The role of self-regulatory processes. Development and Psychopathology, $15,139-162$.

[20] Egeland, B., Carlson, E., \& Sroufe, L. A. (1993). Resilience as process. Development and psychopathology, 5, 517-528.

[21] Veselska, Z., Geckova, A. M., Orosova, O., Gajdosova, B., van Dijk, J. P., \& Reijneveld, S. A. (2009). Self-esteem and resilience: The connection with risky behavior among adolescents. Addictive Behaviors, 34, 287-291.

[22] Jindal-Snape, D., \& Miller, D. J. (2008). A challenge of living? Understanding the psycho-social processes of the child during primary-secondary transition through resilience and self-esteem theories. Educational Psychology Review, 20, 217-236.

[23] Bonanno, G. A. (2004). Loss, trauma, and human resilience: have we underestimated the human capacity to thrive after extremely aversive events? American psychologist, 59, 20-28.

[24] Benetti, C., \& Kambouropoulos, N. (2006). Affect-regulated indirect effects of trait anxiety and trait resilience on self-esteem. Personality and individual differences, 41, 341-352.

[25] Kidd, S. \& Shaharr, G. (2008). Resilience in homeless youth: The key role of self-esteem. American Journal of Orthopsychiatry, 78, 163-172.

[26] Dumont, M., \& Provost, M. A. (1999). Resilience in adolescents: Protective role of social support, coping strategies, self-esteem, and social activities on experience of stress and depression. Journal of youth and adolescence, 28, 343-363.

[27] Tsuzuki, M. (2006). Development of self concept in junior high school students. Kyoikugakuronshu, 48, 363-377 (in Japanese).

[28] Harter, S. (1982). The perceived competence scale for children. Child Psychology, 53, 87-97.

[29] Sakurai, S. (1983). Ninchisareta konpitensushakudo (Nihongoban) no sakusei. The Japanese Journal of Educational Psychology, 31, 245-249 (in Japanese).

[30] Ishige, M. (2004). Relation between resilience and apathy in junior high school students. Journal of the Graduate School of Humanities and Science, 6, 243-252 (in Japanese). 
[31] Schermelleh-Engel, K., Moosbrugger, H., \& Müller, H. (2003). Evaluating the fit of structural equation models: Test of significance and descriptive goodness-of-fit measures. Methods of Psychological Research Online, 8, 23-74.

[32] Kasho, M. (2012). Time perspective and motivation in interpersonal contexts. Japanese Psychological Research, 54, 297-309.

[33] Ridgeway, C. L. (2001). Gender, status, and leadership. Journal of Social issues, 57, 637-655.

[34] Wagner, D. G., \& Berger, J. (1997). Gender and interpersonal task behaviors: Status expectation accounts. Sociological Perspectives, 40, 1-32.

[35] Takagishi, Y., Sakata, M., \& Kitamura, T. (2011). Effects of Self-Esteem on State and Trait Components of Interpersonal Dependency and Depression in the Workplace. Journal of Clinical Psychology, 67, 1-9.

[36] Daniel, M., Brown, A., Dhurrkay, J., Cargo, M. D., \& O'Dea, K (2006). Mastery, perceived stress and health-related behavior in northeast Arnhem Land: a cross-sectional study. International
Journal for Equity in Health, 5, 1-10.

[37] Kling, K. C., Hyde, J. S., Showers, C. J., \& Buswell, B. N. (1999). Gender differences in self-esteem: a meta-analysis. Psychological bulletin, 125, 470-500.

[38] Bolognini, M., Plancherel, B., Bettschart, W., \& Halfon, O. (1996). Self-esteem and mental health in early adolescence: Development and gender differences. Journal of adolescence, $19,233-245$.

[39] Rosenberg, M. (1962). The association between self-esteem and anxiety. Journal of Psychiatric Research, 1, 135-152.

[40] Dolan, S. L. (2007). Stress, self-esteem, health and work. New York: Palgrave Macmillan.

[41] Kessler, R. C., Brown, R. L., \& Broman, C. L. (1981). Sex differences in psychiatric help-seeking: evidence from four large-scale surveys. Journal of health and social behavior, 22, 49-64. 